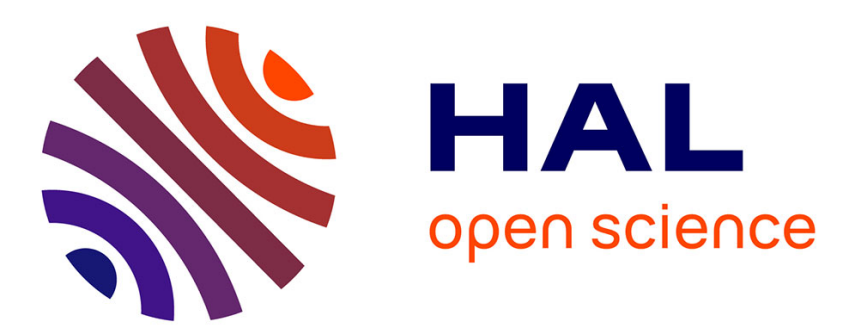

\title{
Applications des supraconducteurs à l'électrotechnique
}

J.P. Chabrerie

\section{To cite this version:}

J.P. Chabrerie. Applications des supraconducteurs à l'électrotechnique. Revue de Physique Appliquée, 1969, 4 (4), pp.565-568. 10.1051/rphysap:0196900404056500 . jpa-00243333

\section{HAL Id: jpa-00243333 https://hal.science/jpa-00243333}

Submitted on 1 Jan 1969

HAL is a multi-disciplinary open access archive for the deposit and dissemination of scientific research documents, whether they are published or not. The documents may come from teaching and research institutions in France or abroad, or from public or private research centers.
L'archive ouverte pluridisciplinaire HAL, est destinée au dépôt et à la diffusion de documents scientifiques de niveau recherche, publiés ou non, émanant des établissements d'enseignement et de recherche français ou étrangers, des laboratoires publics ou privés. 


\title{
APPLIGATIONS DES SUPRAGONDUGTEURS A L'ÉLEGTROTEGHNIQUE
}

\author{
J. P. GHABRERIE, \\ Laboratoire Central des Industries Électriques et Laboratoire de Génie Électrique ${ }^{(1)}$.
}

\begin{abstract}
Résumé. - Après un recensement des nombreuses applications des supraconducteurs dans des domaines tels que : transport d'énergie, stockage d'énergie, conversion des énergies, seule l'application des supraconducteurs aux machines tournantes fait l'objet d'un développement. Les possibilités de réalisation de machines hétéropolaires sont envisagées et deux types de machines homopolaires supraconductrices, actuellement en cours de construction (machine I.R.D. et machine L.C.I.E.), sont décrits.
\end{abstract}

Les développements et l'orientation actuelle des recherches sont examinés.

\begin{abstract}
After a quick survey of the various applications of superconductors in such field as energy transport, energy storage and energy conversion, only applications of superconductivity to rotating machinery are considered. The feasibility of heteropolar engines is examined, and two kinds of superconducting homopolar engines being built at the present time (I.R.D. and L.C.I.E. engines) are described.

Developments and current directions of research in this field are summarized.
\end{abstract}

I. Introduction. - Les applications des supraconducteurs à l'électrotechnique peuvent être classées suivant trois domaines fondamentaux qui sont :

Le transport d'énergie. - Il existe de nombreux projets et brevets de cryoliaisons supraconductrices fonctionnant en continu ou en alternatif.

Le stockage d'énergie. - Des travaux ont été menés à bien dans ce domaine, tendant à définir la forme du bobinage supraconducteur, la mieux adaptée, compte tenu des propriétés supraconductrices et mécaniques des matériaux utilisés.

On pourrait inclure dans ce domaine les multiples applications des électroaimants supraconducteurs.

La conversion des énergies. - Dans ce domaine, les principales applications étudiées sont :

a) les pompes à flux;

b) les redresseurs;

c) les transformateurs;

d) les machines tournantes.

Nous allons examiner plus particulièrement les incidences de l'emploi des supraconducteurs sur le rendement, la puissance volumique ou la puissance massique des machines tournantes. C'est en effet un des domaines où le plus grand nombre de projets ont abouti ou vont aboutir.

II. Généralités. - On peut classer les machines tournantes en deux grandes catégories :

II.1. LES MAGHINES HÉTÉROPOLAIRES dans lesquelles un volume élémentaire de conducteur d'induit est

(1) Associé au C.N.R.S.

REVUE DE PHYSIQUE APPLIQUÉE. - T. 4. No 4. DÉCEMBRE 1969. soumis à un champ inducteur variable au cours d'une révolution du rotor (exemples : turboalternateur, moteur asynchrone, etc.).

Les dissipations thermiques élevées des supraconducteurs en champ alternatif fort font que l'on ne doit pas songer à l'élaboration de machines asynchrones. Le seul type de machine hétéropolaire envisagé par la suite sera une machine synchrone, à induit mobile polyphasé, et inducteur fixe (quadrupôle supraconducteur par exemple).

II.2. LeS MAGHines homopolaires oU UN ÉLÉMENT D'INDUIT EST SOUMIS A UN GHAMP INDUGTEUR CONSTANT LORS D'UNE RÉVOLUTION GOMPLÈTE DU ROTOR. - Il est commode d'exprimer la puissance électromagnétique d'une machine hétéropolaire sous la forme [5] :

$$
\overline{\mathbf{P}}=K_{\mathrm{m}} U_{\mathrm{m}} B_{\mathrm{m}} J_{\mathrm{m}} V \cos \varphi
$$

celle d'une machine homopolaire s'écrivant :

$$
P=K_{\mathrm{m}} U_{\mathrm{m}} B_{\mathrm{m}} J_{\mathrm{m}} V
$$

- $U_{\mathrm{m}}, B_{\mathrm{m}}, J_{\mathrm{m}}$ représentent respectivement les valeurs maximales de la vitesse, de l'induction et de la densité de courant effectivement réalisées dans l'induit de volume $V$,

- $\varphi$ est l'angle de déphasage du courant de ligne par rapport à la tension simple étoilée du système polyphasé induit,

- $K_{\mathrm{m}}$ est appelé " coefficient morphologique d'induit». Il ne dépend, pour un type de machine déterminé, que de la morphologie de la machine : structure interne de l'induit, topographie du champ inducteur, etc. Il reste pratiquement invariant lorsqu'on fait subir à un type de machine donné une homothétie ayant pour centre le centre de symétrie de la machine. 
III. Intérêt de l'introduction des supraconducteurs dans la réalisation de machines tournantes. III.1. MAGHINES hÉTÉROPOlAIRES. - Les valeurs habituellement rencontrées dans les gros alternateurs classiques sont :

$$
\begin{aligned}
J_{\mathrm{m}} & \simeq 5 \times 10^{6} \text { à } 10^{7} \mathrm{~A} \cdot \mathrm{m}^{-2} \\
B_{\mathrm{m}} & \simeq 2 \mathrm{~Wb} \cdot \mathrm{m}^{-2} \\
U_{\mathrm{m}} & \simeq 200 \mathrm{~m} \cdot \mathrm{s}^{-1} \\
K_{\mathrm{m}} & \simeq 0,03 \text { à } 0,05 .
\end{aligned}
$$

Pour la machine synchrone définie plus haut, il serait erroné de penser que l'utilisation d'un inducteur supraconducteur conduise simplement à un gain de plusieurs unités sur la valeur de $B_{\mathrm{m}}$ et à un gain de l'ordre de 2 sur $K_{\mathrm{m}}$ (l'absence de fer permettant une meilleure utilisation du volume utile d'induit).

En effet, des problèmes nouveaux apparaissent :

- Problèmes de réalisation mécanique de l'induit, et de transfert de la puissance au réseau.

- Problèmes de refroidissement liés à l'apparition de pertes par courant de Foucault très notablement supérieures à celles qui se produisent dans les machines classiques.

- Problèmes de réalisation de l'inducteur supraconducteur soumis au couple de réaction de la machine.

Signalons en outre que, lors des variations de charge de la machine, des pertes peuvent apparaître dans l'inducteur supraconducteur soumis alors à un champ magnétique variable.

L'amélioration de la puissance volumique et du rendement serait effective à condition d'élaborer de nouvelles structures d'induit, utilisant par exemple des conducteurs élémentaires très fins, permettant d'évacuer des pertes importantes, nécessitant par-là des systèmes de refroidissement efficaces.

Il est cependant à noter que le programme de la conférence de Londres du 24 au 26 mars 1969 sur «Low Temperatures and Electric Power» ne comporte aucune communication sur ce type de machines [4].

III.2. MAchines HOMOPOLAIRES. - L'expression $P / V$ de la puissance volumique [2] permet de constater immédiatement que la puissance volumique d'une machine homopolaire à inducteur supraconducteur est importante du fait :

- de l'absence de circuit magnétique, ce qui permet de réaliser le rotor en matériau bon conducteur (cuivre, aluminium, etc.), $J_{\mathrm{m}}$ pouvant atteindre en régime continu de $5 \times 10^{6}$ à $10^{7} \mathrm{~A} \cdot \mathrm{m}^{-2}$,

- des valeurs de $B_{\mathrm{m}}$ (plusieurs $\mathrm{Wb} . \mathrm{m}^{-2}$ ) que l'on peut obtenir en utilisant des supraconducteurs,

- des valeurs élevées de $K_{\mathrm{m}}$ caractérisant les machines homopolaires $\left(K_{\mathrm{m}} \simeq 0,2\right)$ [5] (absence de ferromagnétiques).
Nous en déduisons donc, ce qui est aussi très important pour les applications, que le couple volumique (obtenu en divisant la puissance volumique par la vitesse angulaire) est aussi très élevé pour les mêmes raisons que précédemment.

Les principales difficultés de réalisation rencontrées sont liées :

- à la détermination des formes optimales de l'induit et l'inducteur,

- à l'utilisation de contacts adaptés à la tension faible et au courant élevé, spécifiques des machines homopolaires.

IV. Les machines homopolaires supraconductrices. - On doit considérer deux types de machines homopolaires :

1) Les machines où la f.e.m. à vide est le résultat de l'intégration de la composante axiale du champ électrique induit.

2) Les machines à disques ou à segments radiaux pour lesquelles la composante radiale de ce champ est mise en œuvre.

Pour des raisons évidentes, seul le système inducteur de ces deux types de machines est réalisé en matériaux supraconducteurs. De plus, la réaction d'induit n'exerce aucun effort sur ce bobinage.

Nous n'envisagerons par la suite que les machines du type « radial» qui n'utilisent qu'un seul inducteur.

Les machines homopolaires sont à basse tension et forte intensité de courant, ce qui nécessite :

- l'emploi d'artifices permettant d'élever la tension d'utilisation (disques ou segments multiples mis en série par des éléments fixes),

- l'utilisation de systèmes collecteurs de courant de hautes performances.

IV.1. Projets et réalisations. - a) Machines I.R.D. - L'International Research and Development (Newcastle/Tyne U.K.) a réalisé un prototype développant $36 \mathrm{~kW}$ à $2000 \mathrm{t} . \mathrm{mn}^{-1}$. La construction d'un modèle de $2400 \mathrm{~kW}$ est pratiquement achevée [6].

Le principe de fonctionnement est donné par les figures 1 et 2 . Les f.e.m. développées entre les extrémités de chacun des segments radiaux constituant le rotor sont mises en série par l'intermédiaire d'un système de conducteurs fixes et de collecteurs de courant utilisant des balais métallographitiques glissants. C'est sur cette partie statique de l'induit que s'exerce le couple de réaction de la machine.

Rappelons que l'emploi de contacts glissants de ce type n'est possible qu'au-delà d'une certaine valeur du produit $N \Phi$ ( $\Phi$ flux inducteur utile en $\mathrm{Wb}, N$ fréquence de rotation en $\left.\mathrm{t} . \mathrm{s}^{-1}\right)$.

- Principales caractéristiques du moteur homopolaire de moyenne puissance I.R.D. dont le fonctionnement est prévu aux environs de juin 1969 (C.F. [4]). Une étude économique menée à bien par cette 


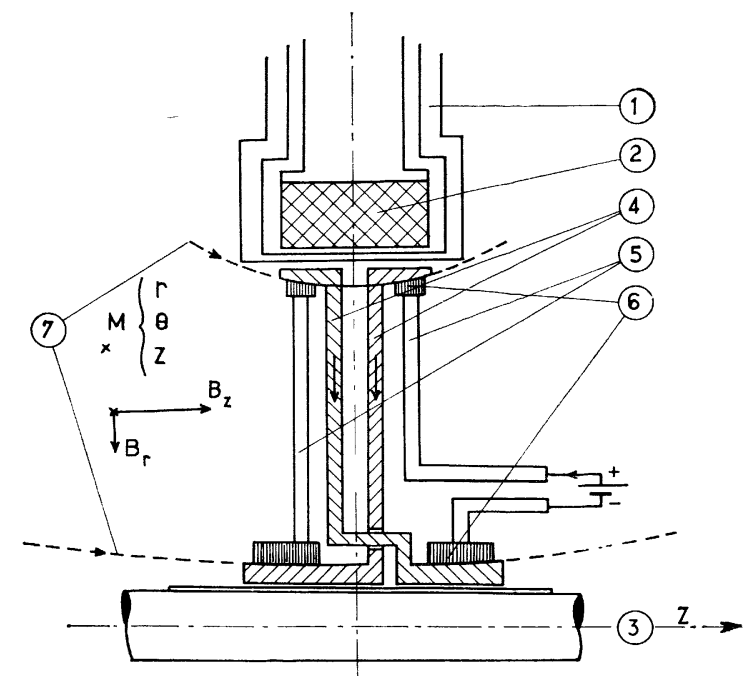

FIG. 1. - Schéma de fonctionnement de la machine homopolaire à inducteur supraconducteur élaborée par l'International Research and Development (Newcastle, G.-B.) :

(1), Appareillage cryogénique ; (2), Bobinage inducteur (solénoïde supraconducteur) ; (3), Axe de rotation ; (4), Segments mobiles du rotor; (5), Segments fixes de 1'induit ; (6), Collecteurs de courant; (7), Lignes de forces du champ magnétique inducteur.

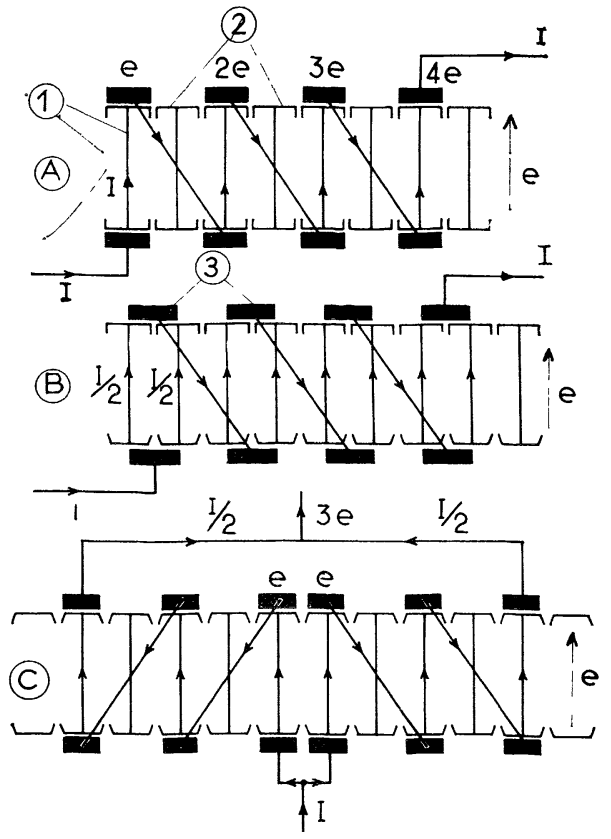

FIG. 2. - Schéma d'interconnexion des segments fixes et mobiles d'induit de la machine homopolaire supraconductrice I.R.D

$2 \mathrm{~A})$ et $2 \mathrm{~B}$ ) Disposition série.

2 C) Disposition série-parallèle permettant de limiter la tension entre deux segments consécutifs quelconques mobiles de l'induit, à la valeur de la tension élémentaire e : e, tension élémentaire aux bornes de chaque segment mobile de l'induit; (1), Segments mobiles d'induit; (2), Contacts glissants ; (3), Contacts fixes. firme a été publiée dans « Electronics and Power » (mars 1968). Elle est rapportée ci-dessous et permet certaines comparaisons entre deux moteurs $8000 \mathrm{CV}$, 50 t.mn.

$\begin{array}{lcc} & \begin{array}{c}\text { Moteur } \\ \text { Conventionnel }\end{array} & \begin{array}{c}\text { Moteur } \\ \text { SUPRACONDUGTEu }\end{array} \\ & - & - \\ \text { Poids } & 370 \text { tonnes } & 40 \text { tonnes } \\ \text { Prix } & £ 220 \times 10^{3} & £ 130 \times 10^{3} \\ \text { Rendement } & 94 \% & 97 \%\end{array}$

b) Moteur homopolaire L.C.I.E. $\left(^{2}\right)$. - Une maquette probatoire est actuellement en cours de fabrication au L.C.I.E. Le principe de fonctionnement du moteur est donné par le schéma de la figure 3.

La méthode utilisée pour accroître la tension d'alimentation est la mise en série des f.e.m. développées aux bornes des contacts central et périphérique de

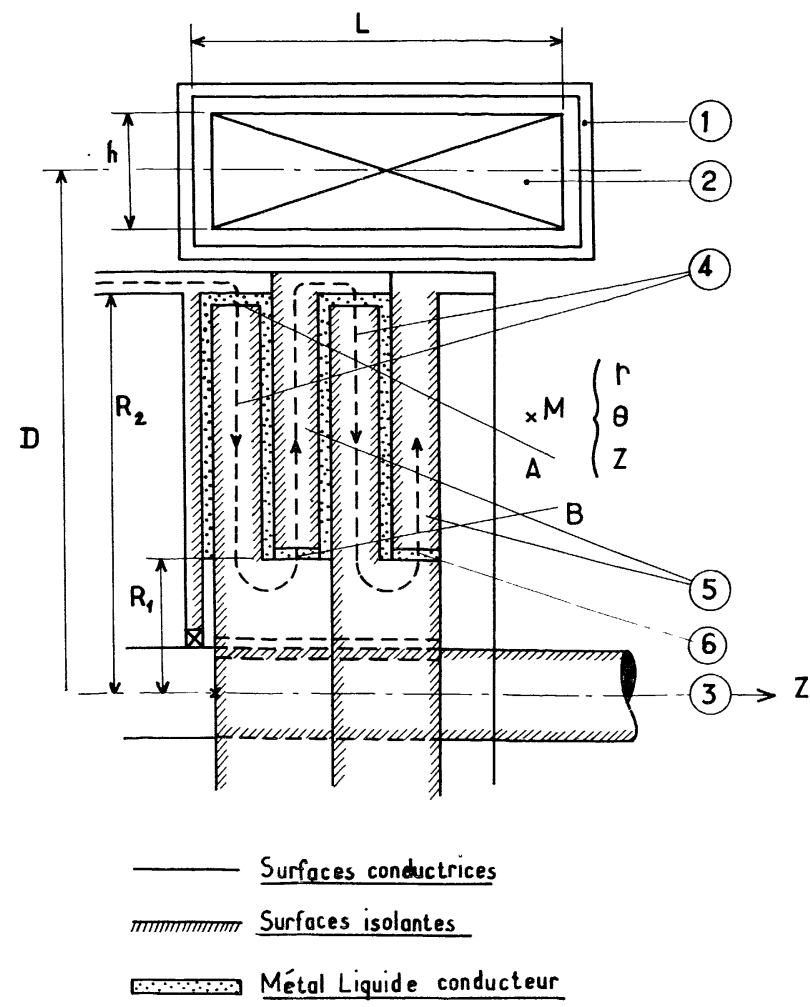

FIG. 3. - Schéma de fonctionnement de la machine homopolaire à disques multiples et inducteur supraconducteur élaborée par le Laboratoire Central des Industries Électriques :

(1), Appareillage cryogénique ; (2), Bobinage inducteur (solénoïde supraconducteur) ; (3), Axe de rotation ; (4), Disques mobiles d'induit ; (5), Contre-disques fixes d'induit; (6), Métal liquide conducteur assurant les contacts électriques.

(2) Etude effectuée grâce au soutien financier de la D.G.R.S.T. et de la D.R.M.E. 
chaque disque mobile par l'intermédiaire de contredisques fixes.

D'excellents contacts sont nécessaires en $\mathrm{A}$ et $\mathrm{B}$ ( fig. 3). Ils sont obtenus avec l'aide d'une méthode de contact particulière qui consiste à remplir l'espace libre entre les différents disques et contre-disques d'induit d'un métal liquide conducteur [11]. Nous utilisons le composé stable défini HgIn [10]. La théorie de cette méthode a été faite dans l'hypothèse d'écoulements laminaires [7] et turbulents [9]. Elle permet de calculer avec une bonne approximation les pertes globales (M.H.D. + Joule) dues à ce mode de contact. Une théorie permettant l'optimisation de ce type de machine a été menée à bien. La puissance volumique maximale, à quantité de matériaux supraconducteurs et vitesse de rotation données, est obtenue pour des inducteurs assez plats.
Conclusion. - Bien que le champ d'application des machines hétéropolaires soit notablement plus étendu que celui des machines homopolaires, c'est dans ce dernier domaine que l'incidence des supraconducteurs sur la réalisation des machines tournantes a fait l'objet des recherches les plus développées. Ceci est dû au fait que des problèmes technologiques importants se posent dans une machine hétéropolaire supraconductrice, limitant par-là l'accroissement du rendement et de la puissance spécifique par rapport aux réalisations classiques, alors que les machines homopolaires représentent une application presque idéale pour les supraconducteurs. Ces derniers permettent, de plus, d'obtenir de hautes performances dans des domaines précis - moteurs à courant continu de grande puissance à basse vitesse par exemple - où l'électrotechnique classique est désavantagée.

\section{BIBLIOGRAPHIE}

[1] BuchHoLd (T. A.), Three-phase motor with superconducting bearings, General Electric Research Laboratory, Report no 65, RL/3881 M, février 1965.

[2] STEKLy (Z. J. J.) et Woodson (H. H.) (AvcoEverett Research Laboratory), Rotating machinery utilizing superconductors, IEEE Transactions on Aevospace, 1964, 2, 826-841.

[3] Woodson (H. H.), STEkLy (Z. J. J.) et HaLas (E.), A study of alternators with superconducting field windings, IEEE Transactions on power apparatus and systems, 1966, PAS-85, 264-280.

[4] Appletion (A. D.), Macnab (R. B.), Tinlin (F.) et Ross (J.S. H.), "Proceedings of the Conference on low temperatures and electric power ", 24 au 26 mars 1969 (Londres U.K.).

[5] MAILFERT (A.), Sur les machines électriques tournantes hétéropolaires sans circuit magnétique, R.G.E., 1967, 76, 2.

[6] International Research and Development Co. Superconducting Motor, "Electronics and Power ", mars 1968, 114.
[7] Chabrerie (J. P.), Fournet (G.) et Matlferit (A.), Étude d'un mode de contact par métaux liquides dans une machine homopolaire, R.G.E., 1968, 77, 279-287.

[8] Bonnefille (R.), Chabrerie (J. P.), Fournet (G.), MAILFERT (A.), RoBERT (J.) et VAILLANT (C.), Machine homopolaire à inducteur supraconducteur, Proceedings of the 2nd International Cryogenic, Engineering Conference, Brighton (U.K.), 710 mai 1968.

[9] Chabrerie (J. P.), MatlFerT (A.) et RoberT (J.), "Filling up " sliding electrical contacts for and homopolar engine. Proceedings of the $1968 \mathrm{Holm}$ Seminar on Electric Contact Phenomena, 11-15 novembre 1968. Illinois Institute of Technology and I.I.T. Research Institute, Chicago (U.S.A.).

[10] ROBERT (J.), Contribution à l'étude physico-chimique des amalgames, applications à l'électrotechnique, R.G.E., 1965, 74, 317.

[11] Poulain (J.), Contribution à l'étude des machines homopolaires à courant continu, Thèse, Paris, juin 1962.

[12] Claude (M. L.), Contribution à l'étude des alternateurs à enroulements supraconducteurs, Thèse, Paris, 1966. 